\title{
A Personal Adventure in Muon-Catalyzed Fusion
}

\author{
John David Jackson*
}

Luis Alvarez and colleagues discovered muon-catalyzed fusion of hydrogen isotopes by chance in late 1956. On sabbatical leave at Princeton University during that year, I read the first public announcement of the discovery at the end of December in that wellknown scientific journal, The New York Times. A nuclear theorist by prior training, I was intrigued enough in the phenomenon to begin some calculations. I describe my work here, my interaction with Alvarez, and a summary of the surprising developments, both before and after Alvarez's discovery. The rare proton-deuteron $(p-d)$ fusion events in Alvarez's liquid-hydrogen bubble chamber occurred only because of the natural presence of a tiny amount of deuterium (heavy hydrogen). Additionally, the fusion rate, once the proton-deuteron-muon $\left(p d \mu^{-}\right)$molecular ion has been formed, is sufficiently slow that only rarely does an additional catalytic act occur. A far different situation occurs for muons stopping in pure deuterium or a deuterium-tritium $(d-t)$ mixture where the fusion rates are many orders of magnitude larger and the molecular-formation rates are large compared to the muon's decay rate. The intricate interplay of atomic, molecular, and nuclear science, together with highly fortuitous accidents in the molecular dynamics and the hope of practical application, breathed life into a seeming curiosity. A small but vigorous worldwide community has explored these myriad phenomena in the past 50 years.

Key words: Luis W. Alvarez; Venedikt P. Dzhelepov; Frederick Charles Frank; Semen S. Gershtein; Leonid I. Ponomarev; Andrei D. Sakharov, Yakov B. Zel'dovich; muonic molecules; muon-catalyzed fusion; fusion; energy production; sticking probability.

The observation by Luis Alvarez (figure 1) and coworkers of the formation of muonic atoms and the subsequent catalysis of nuclear fusion in a liquid-hydrogen bubble chamber in late 1956 was entirely accidental, totally unexpected, and peripheral to the group's main study of hadronic interactions. ${ }^{1}$ From the point of view of fundamental physics, the phenomena are completely "understood." As I comment in more detail below, Frederick Charles Frank had addressed the concepts briefly in $1947,{ }^{2}$ as did Andrei D. Sakharov in $1948,{ }^{3}$ and Yakov

\footnotetext{
* John David Jackson is Professor Emeritus of Physics at the University of California, Berkeley, where he has been since 1967. His interests lie in theory close to experiment, and recently in some corners of the history of science.
} 


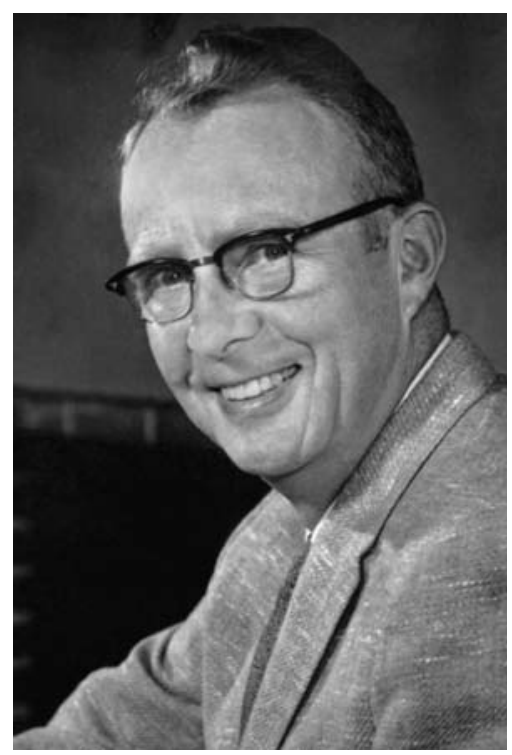

Fig. 1. Luis W. Alvarez (1911-1988). Credit: Lawrence Berkeley National Laboratory.

B. Zel'dovich in 1954. ${ }^{4}$ Before 1957, Frank's note and Zel'dovich's paper in Russian went largely unnoticed; Sakharov's unpublished report was known only inside the Soviet nuclear-weapons establishment. The experimental discovery was viewed as exciting and bizarre. It led immediately to speculation on energy production by "cold fusion." 5

I know exactly when and where I first became aware of muon-catalyzed fusion. During the academic year 1956-1957, I was visiting the Physics Department at Princeton University on a John Simon Guggenheim Memorial Fellowship. That fall I thought about a few problems in nuclear physics but had not settled into any serious research. By early December there were rumors about parity nonconservation in the experiments of Chien-Shiung $\mathrm{Wu}$ and coworkers and also rumors of a second mu-like meson $\left(\mu^{\prime}\right)$ at Berkeley but nothing definite, and by the time of the long Christmas vacation Palmer Laboratory was virtually deserted. Now, one of the virtues of being within 100 miles of New York City in those days was the delivery of The New York Times to one's doorstep each morning. On the morning of December 29, 1956, I read a report of a paper presented the previous day at an American Physical Society meeting in Monterey, California. ${ }^{5}$ My yellowed clipping from The Times is displayed in figure 2. It reported the discovery by the Alvarez group of the muon catalysis of proton-deuteron $(p-d)$ fusion in a liquid-hydrogen bubble 


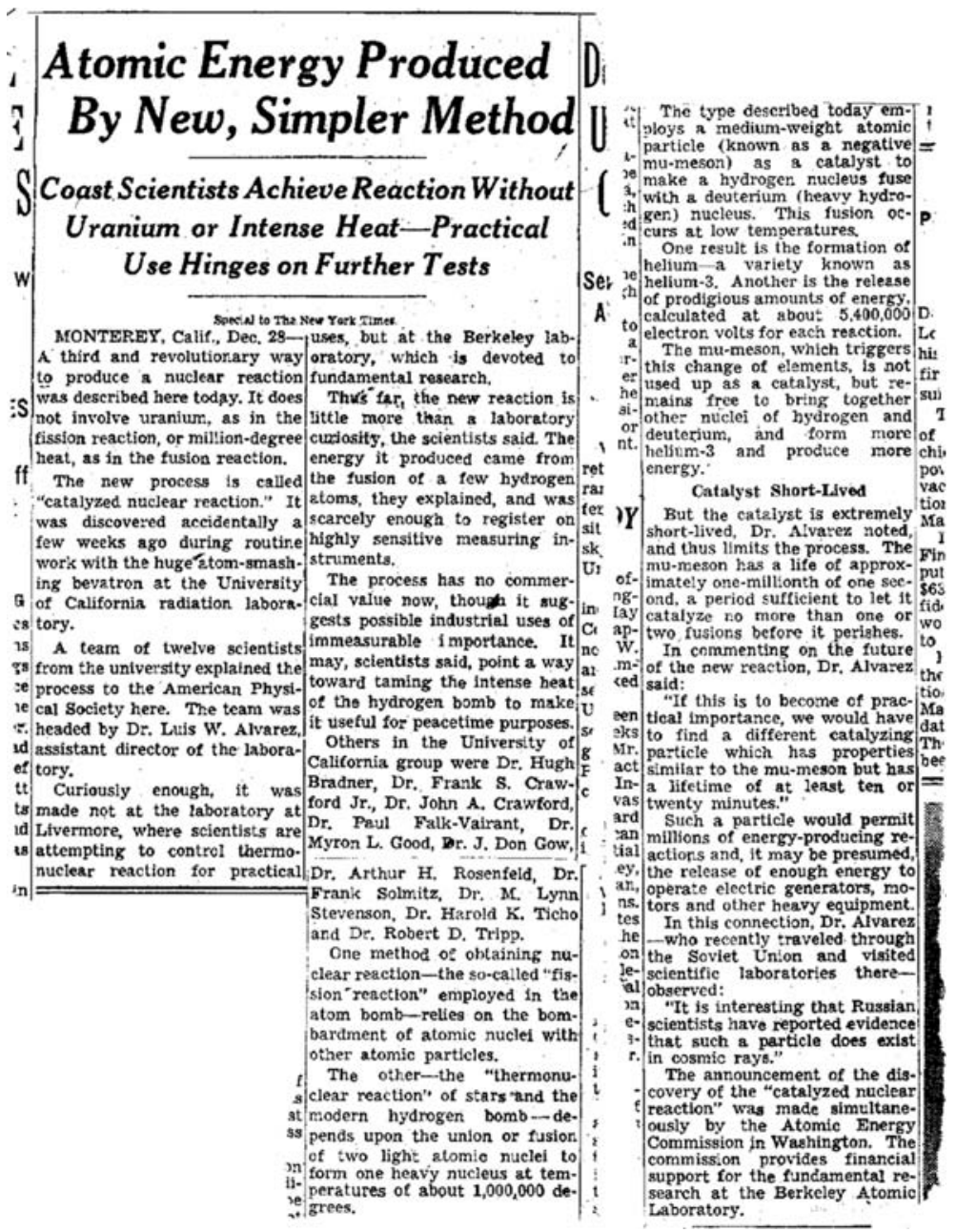

Fig. 2. Reproduction of a cutting from The New York Times of December 29, 1956, reporting the announcement at Monterey on the previous day of the discovery of the muon-catalyzed fusion of hydrogen isotopes. Credit: Reproduced by permission of The New York Times, 229 West 43rd Street, New York, NY 10036.

chamber. The process was contrasted with "hot" fusion. Alvarez speculated on possible energy production. I quote the headline and a few lines from the story: 


\section{Atomic Energy Produced By New, Simpler Method Coast Scientists Achieve Reaction Without Uranium or Intense Heat: Practical Use Hinges on Further Tests}

The new process is called "catalyzed nuclear reaction."...

The process has no commercial value now, though it suggests possible industrial uses of immeasurable importance....

But the catalyst is extremely short-lived, Dr. Alvarez noted, and thus limits the process....

"If this is to become of practical importance, we would have to find a different catalyzing particle which has properties similar to the mu-meson but has a lifetime of at least ten or twenty minutes.”...

In this connection, Dr. Alvarez ... observed:

"It is interesting that Russian scientists have reported evidence that such a particle does exist in the cosmic rays." 6

My imagination was stirred by the newspaper article, and I started immediately to play with the physics of the process. I could work uninterrupted in the deserted lab and rapidly calculated-with slide rule and graph paper for integrations of the barrier penetrations and evaluation of matrix elements!

The catalytic action of a negative muon $\left(\mu^{-}\right)$in a liquid-hydrogen bubble chamber is a multistep process, beginning with the muon entering the liquid with a kinetic energy of the order of several $\mathrm{MeV}$ (million electron volts). A crucial time scale is set by the muon's mean life of $\tau=2.2 \times 10^{-6} \mathrm{~s}$ (second). In 1950 Arthur Wightman showed that a muon in liquid hydrogen will slow down, be captured by one of the nuclei in a hydrogen molecule, and cascade down to its own atomic ground state in roughly $10^{-9} \mathrm{~s}^{7}$ The muon-hydrogenic atom is 200 times smaller than an ordinary hydrogen atom and is an electrically neutral object, a pseudo-neutron, $11 \%$ heavier than a proton when viewed on ordinary atomic scales. It will escape and move at thermal speeds, making numerous collisions with the hydrogen nuclei. In normal hydrogen, those collisions are mostly with protons, only rarely with the tiny fraction $(1 / 6,000)$ of naturally occurring deuterons present. But such collisions are crucial. Edward Teller, in a bravura performance on hearing about the results, pointed out that the reducedmass effect makes the muon's binding energy to the deuteron $(d)$ greater than to a proton $(p)$; the charge transfer reaction $\left(\mu^{-} p\right)+d \rightarrow p+\left(\mu^{-} d\right)$ is exoergic by energy $Q=135 \mathrm{eV}$ with an inverse-velocity $(1 / v)$ cross section. The muon jumps from the proton to the deuteron and then goes on eventually to find a proton to form a $\left(p^{+} d^{+} \mu^{-}\right)$molecular ion that undergoes the catalytic reaction $\left(p^{+} d^{+} \mu^{-}\right)$ $\rightarrow{ }^{3} \mathrm{He}+\mu^{-}+5.5 \mathrm{MeV}$. The reduced-mass effect is the only reason why the Alvarez group observed even a small number of catalyzed reactions among the many muon decays. In pure deuterium or a deuterium-tritium $(d-t)$ mixture, the reduced-mass effect is of minor importance; reactive mu-mesic molecular ions form as a matter of course. 
Once a molecular ion is formed in a low-lying energy state, the system undergoes the customary molecular dynamics of vibration and rotation, with the nuclei kept apart by the repulsive Coulomb barrier. The vibration times are of the order of $5 \times 10^{-18} \mathrm{~s}$. The muon orbits roughly ten times faster around the "slow-moving" nuclei in the adiabatic (Born-Oppenheimer) approximation. In electronic molecules the probability of the nuclei to vibrate to close distances (and thus fuse) is totally negligible, but the much larger muon mass reduces the distances by a factor of 200. Barrier penetration to zero separation is still small, but finite.

The conversion of the nuclear-reaction cross section $\sigma$ to a reaction rate for a given molecular ion proceeds as follows: At extremely low energies, an exoergic cross section for charged particles follows a "Gamow" form, $\sigma=A C^{2} / v$, where $A$ is the reaction constant (in $\left.\mathrm{cm}^{3} / \mathrm{s}\right), v$ is the relative velocity $(\mathrm{cm} / \mathrm{s})$ in the collision, and $[\mathrm{C}(v)]^{2}$ is the s-wave Coulomb-penetration factor. The experimental data on the cross section at low energies can be used to find $A$. The reaction rate $\lambda_{r}$ within the molecular ion is given by the formula, $\lambda_{r}=A|\psi(0)|^{2}$, where $\psi(0)$ is the wave function of the molecular ion at zero separation of the nuclei (actually, at nuclear distances).

Since the catalytic muon responds more or less instantly to the "slow" nuclear motions, at the moment just before the nuclear reaction occurs, the muon "sees" a stationary helium nucleus of charge $Z=2$, and its wave function will be that of a muonic helium atom.

The nuclear reactions of interest are as follows:

$$
\begin{array}{ccc}
p+d & \rightarrow{ }^{3} \mathrm{He}+\gamma, & Q=5.5 \mathrm{MeV} \\
d+d & \rightarrow{ }^{3} \mathrm{He}+n, & Q=3.3 \mathrm{MeV} \\
d+d & \rightarrow \quad t+p, & Q=4.0 \mathrm{MeV} \\
d+t \rightarrow & \rightarrow{ }^{4} \mathrm{He}+n, & Q=17.6 \mathrm{MeV}
\end{array}
$$

It is understood that on the left-hand side of all four catalytic versions of the reactions a negative muon binds the hydrogen isotopes into a molecular ion $\left(p^{+} d^{+} \mu^{-}, d^{+} d^{+} \mu^{-}\right.$, or $\left.p^{+} t^{+} \mu^{-}\right)$. On the right-hand side, in the first reaction the gamma ray $(\gamma)$ is most often replaced by a muon, but sometimes a muon and a gamma ray. In the other three reactions, a freed catalytic muon is understood on the right-hand side in addition to the nuclear particles. Because of my background in nuclear physics, I started out on the nuclear reaction rates by first finding the cross-section data. With the potential-energy curves for the hydrogen molecular ion from the literature and WKB (Wentzel-Kramers-Brillouin) approximations for the extrapolation of the vibrational molecular wave functions to zero nuclear separation, using my slide rule and graph paper I found the spin-averaged total nuclear reaction rates from the molecular ground states to be*

\footnotetext{
* More recent data on the $p-d$ reaction cross section makes the rate a factor of four larger, approximately equal to the muon's decay rate. Apart from anything else, this explains why the Alvarez group found only two examples of a second catalysis by a muon.
} 


$$
\begin{aligned}
& \lambda_{r}(p d \mu) \approx 1.8 \times 10^{5} \mathrm{~s}^{-1} \\
& \lambda_{r}(d d \mu) \approx 3.5 \times 10^{10} \mathrm{~s}^{-1} \\
& \lambda_{r}(d t \mu) \approx 1.1 \times 10^{12} \mathrm{~s}^{-1} .
\end{aligned}
$$

Apart from the $p d \mu$ rate, once the muonic molecular ion has settled to its ground state, the fusion reaction occurs extremely rapidly on the time scale of the muon's lifetime.

The next step in the catalytic cycle is the liberation of the muon together with the nuclear fragments. In the two-body final state of $p d \mu \rightarrow{ }^{3} \mathrm{He}+\mu$, the muon carries off almost all of the $5.5 \mathrm{MeV}$ energy release. In the $d d \mu$ and $d t \mu$ fusions, the final state consists of three bodies - the nuclear fragments share the $Q$ value (3.3, 4.0 , or $17.6 \mathrm{MeV})$; the muon interacts only electromagnetically with the recoiling fragments. But that electromagnetic interaction is crucial in determining the sticking probability $\omega_{s}$, which limits the average number of catalytic events a muon can induce, regardless of how rapidly the catalytic cycle occurs.

The physical picture is as follows: As I remarked earlier, at the instant before fusion occurs the muon finds itself in a hydrogen-like ground state of a $Z=2$ muonic atom. At the instant after, it "sees" either two $Z=1$ nuclei flying apart $(d d \mu \rightarrow t+p+\mu)$ or a $Z=2$ nucleus flying off $\left(d d \mu \rightarrow{ }^{3} \mathrm{He}+n+\mu\right.$ or $\left.d t \mu \rightarrow{ }^{4} \mathrm{He}+n+\mu\right)$. In the latter two cases, the muon has a finite chance of being captured into a bound state around the moving helium nucleus. The calculation of the initial "sticking probabilities" is a simple application of the sudden perturbation of quantum mechanics. ${ }^{8}$ Let the initial wave function be $\langle\boldsymbol{r} \mid 1 s\rangle=\psi_{1 s}(\boldsymbol{r})$ and the final wave function around the moving helium nucleus be $\langle\boldsymbol{r} \mid n\rangle=\psi_{n}(\boldsymbol{r}) \exp (i \boldsymbol{k}$. $\boldsymbol{r}$ ), where $\boldsymbol{r}$ is the muon's coordinate relative to the nucleus and $\boldsymbol{k}=m_{\mu} \boldsymbol{v} / \hbar$, with $\boldsymbol{v}$ the velocity of the helium nucleus. The amplitude for capture of the muon into the moving bound state $n$ is $\langle n \mid 1 s\rangle$ and the initial sticking probability is $\omega_{s}^{0}=|\langle n \mid 1 s\rangle|^{2}$. The simple overlap integrals for capture into the ground and bound excited states yield initial sticking probabilities into all bound states (in the Born-Oppenheimer and sudden perturbation approximations):

$$
\omega_{s}^{0} \approx 1.2 \%\left(d t \mu \rightarrow{ }^{4} \mathrm{He}+n+\mu\right) \text { and } \omega_{s}^{0} \approx 16 \%\left(d d \mu \rightarrow{ }^{3} \mathrm{He}+n+\mu\right) .
$$

The story continues. The muon might yet be "reactivated." The helium nucleus with its bound muon (acting as a $Z=1$ "nucleus") loses energy by inelastic collisions with the atoms of the liquid hydrogen. There is a finite probability that the muonic atom will be ionized as it slows down to thermal energies. I used scaled experimental data on energy loss and ionization, with a single-step approximation (now known to be applicable only at low density) to find the reactivation fraction $R$ to be

$$
\begin{gathered}
R \approx 0.22(d t \mu) \\
R \approx 0.04(d d \mu) .
\end{gathered}
$$


Though not written down explicitly in my paper, these numbers led to final sticking probabilities:

$$
\begin{aligned}
& \omega_{s} \approx 0.94 \%\left(d t \mu \rightarrow{ }^{4} \mathrm{He}+n+\mu\right) \\
& \omega_{s} \approx 15 \%\left(d d \mu \rightarrow{ }^{3} \mathrm{He}+n+\mu\right) \\
& \omega_{s} \approx 8 \%\left(d d \mu \rightarrow{ }^{3} \mathrm{He}+n+\mu \text { and } d d \mu \rightarrow t+n+\mu\right) .
\end{aligned}
$$

Once the muon is bound around a helium nucleus at thermal energies in the liquid hydrogen, it is lost forever, because its binding energy is four times that of a muonic hydrogen atom. The final sticking probabilities show that, however fast the cycling occurs, on average a muon will not initiate more than 100 catalytic acts in a $d-t$ mixture and not more than a dozen in pure liquid deuterium.

A topic I largely neglected was the rate of molecular-ion formation by collisions of the neutral muonic atom with hydrogen nuclei. A serendipitous estimate of $\lambda_{\text {mol }}$ form $\approx 10^{8} \mathrm{~s}^{-1}$ was based on a hasty and totally wrong calculation using the BornOppenheimer approximation that ignored the orthogonality of bound and continuum wave functions. The resulting large overlap integral accidentally simulated the near-resonant conditions of a proper calculation (done much later by others) with muonic molecular states bound by only one or two eV and scattering resonances above threshold.

Because of Alvarez's stress and of that of The New York Times on muoncatalyzed fusion as a potential new source of energy, I made some speculations on energy production based on a maximum of $100 d t$ fusions per muon. With very rough estimates of accelerator efficiencies, pion production, and other quantities, I estimated the energy cost per muon at $10 \mathrm{GeV}$ (giga electron volts) from the electrical mains. With only $1.7 \mathrm{GeV}$ produced by $100 d t$ fusions, my estimate missed the breakeven point by a factor of 5, not including further inefficiencies in the conversion of heat to electrical energy. Conjectures about the maximum capabilities of accelerators led to the view that, even if better than breakeven could be achieved, a purely $\mu \mathrm{CF}$ (Muon-Catalyzed Fusion) power plant would be limited in size to tens of MW (megawatts). In 50 years quite a lot has changed in accelerator technology, and better calculations of sticking probabilities have been made, but I believe my rough considerations are still valid.

Perhaps the most important conclusions of my work were that in the energetically interesting case of the fusion of deuterons with tritons, the nuclear reaction rate once the molecular ion is formed is extremely fast $\left(\lambda \geq 10^{12} \mathrm{~s}^{-1}\right)$; and that, whatever the rates of molecular processes, there is an upper limit of the order of $10^{2}$ on the number of possible fusions caused by the muon because of capture by the produced helium ion, depending only on the velocity of the ion and independent of the muon's lifetime or mass. This last conclusion vitiated the remarks attributed to Alvarez at the end of the news story about the efficacy of a possible longer-lived lepton. 
I must have worked feverishly, for my files show that my paper, "Catalysis of Nuclear Reactions between Hydrogen Isotopes by $\mu^{-}$Mesons," typed by me, was sent to the Physical Review on January 9, 1957, and appeared in April 1957. ${ }^{9}$ My files also contain a carbon copy of a letter I wrote to Alvarez, dated January 5, 1957, that said in part:

Your $\mu^{\prime}$ meson and its explanation were featured in the newspapers of this area around the New Year. Having nothing better to do, I began playing with the problem. The enclosed rough draft is the result (excuse the typing in the paper-it's my own). It is more than likely that you and your group have done similar and better calculations already. I found it entertaining, anyway, to see what could be done from first principles in the way of estimating the various rates. The speculations on power production are, of course, very wild and probably wrong.

Alvarez replied on January 8-note the efficiency of transcontinental postal service in 1957! I quote his letter (figure 3) in its entirety.

\section{Dear Dr. Jackson:}

It was good of you to send us your very interesting report. My theoretical friends are fighting over it at the moment. We are trying to estimate the number of $\mu^{-}$s which get stuck on the recoil $\mathrm{He}^{3}$, after a D $+\mathrm{D} \rightarrow \mathrm{He}^{3}+\mathrm{n}$ reaction. If the $\mu$ isn't stripped in the recoil, it can't get away. If the stripping cross-section is high enough, we'll look for a big burst of neutrons from a Dewar flask of $\mathrm{D}_{2}$ above the large scintillator tank of the Reines-Cowan type, which is here in Berkeley. We had thought of such experiments before, but never could see how the chains would be long enough to make it interesting.

We have a large group of molecular experts now working hard on the whole problem. Teller and [Edwin] McMillan have been thinking hard since the earliest days of the effect and they have been joined by a bunch of younger people. Much of their work parallels yours and I will let them have the fun of communicating their results directly to you, rather than trying to interpret what they have done.

I am enclosing a preprint with a few minor changes.

I have only one request and that is that you change the reference from the New York Times, ${ }^{*}$ to the APS meeting-Monterey. Dec. 28, 1956 and UCRL 3620. The lab is a bit sensitive on this point, since in the case of the antiproton, we received lots of letters from people who thought we held the thing too long before publishing, \& from others who said "Don't you guys publish anywhere except the New York Times?" So in the $\mu$-catalysis, we were careful to follow the book-we presented the thing first at the APS meeting, after sending out

\footnotetext{
* The first reference in my manuscript read: L.W. Alvarez et al., New York Times CVI No.
} 36, B4, 1 (December 29, 1956). 


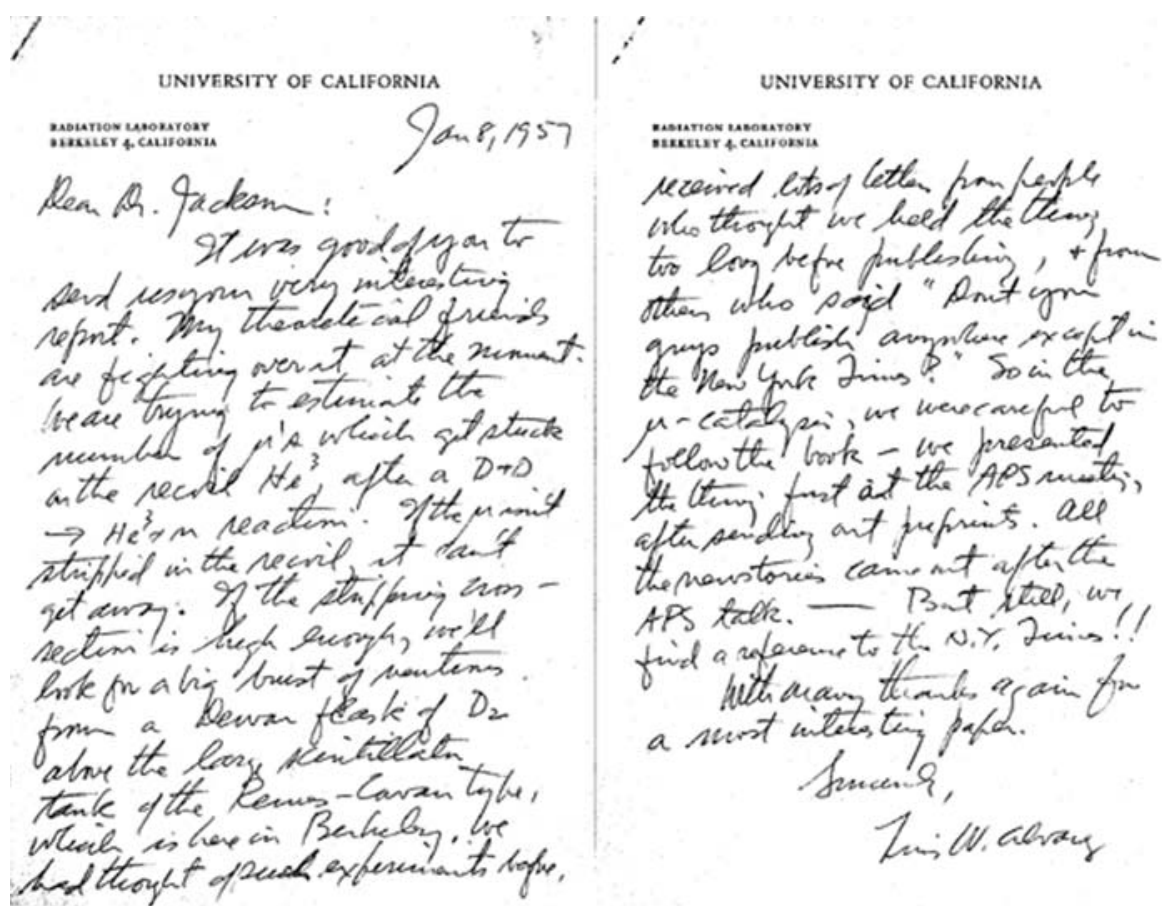

Fig. 3. Pages 1 and 3 of Luis W. Alvarez's three-page handwritten letter to the author of January $8,1957$.

preprints. All the news stories came out after the APS talk.-But still, we find a reference to the N.Y. Times!!

With many thanks again for a most interesting paper.

Sincerely,

Luis W. Alvarez

This was my first contact with Luis Alvarez and it soon led to contacts with members of his group, contacts that have flourished over the years. A not totally frivolous speculation: On what university faculty might I be now if I had not read The New York Times that December day?

Alvarez tells the delightful story of the discovery of the muon-catalysis events and their explanation in his Nobel Prize Lecture. ${ }^{10}$ In those early days, there were no professional bubble-chamber film scanners. The physicists did it themselves. As Luie noted, the physicists' involvement at the scanning level was crucial for the discovery. ${ }^{11}$ Casual instructions to scanners about $\pi-\mu$ (pion-muon) decays would not have triggered the selection of the catalysis events for further study, if only because no one dreamt of their existence. In scanning film from the 10 -inch 
liquid-hydrogen bubble chamber for strange-particle events, the physicists noticed a few anomalously energetic muons. The hypothesis of pion decay in flight (just before stopping) had to be rejected when it was found that the muons all had a kinetic energy of $5.4 \mathrm{MeV}$. The particle physicists needed an astrophysicist colleague to identify the likely source of 5.4-MeV muons, namely, the fusion reaction, $p+d \rightarrow{ }^{3} \mathrm{He}+\gamma$, with the muon being responsible for the union of the proton and deuteron in a molecular ion $(p \mu d)$ and for sometimes carrying off the energy release, instead of the gamma ray.

Neither the physicists in Berkeley nor I in Princeton were aware of considerably earlier theoretical work on the subject until after submission of our papers for publication.

As is well known by now, Frederick Charles Frank (later Sir Charles) gave the first published mention of muon-catalyzed fusion more than 9 years before its experimental observation. ${ }^{12}$ A solid-state physicist and colleague of Cecil F. Powell's at the University of Bristol, Frank examined and rejected a large number of possible alternative explanations of the $\pi-\mu$ decay events that César M.G. Lattes, Giuseppe P.S. Occhialini, and Powell had discovered. ${ }^{13}$ One of the alternatives was the formation of muonic molecular ions, among them $p \mu d$, with its fusion energy release of $5.5 \mathrm{MeV}$. Frank rejected the mechanism because of insufficient deuterium in the emulsion and the incorrect $Q$ value but discussed briefly the formation of $p \mu$ atoms and barrier penetration within the molecule. Andrei Sakharov apparently made the next research on the subject in 1948, which was the basis of his now-legendary unpublished report. ${ }^{14}$ In his own words:

Having become acquainted with a paper of Frisch [sic] in which he discussed a possible alternative interpretation of the experiments of Powell, Lattes, and Occhialini (discovery of the $\pi$ meson) by means of a $\mu$-catalysis reaction, I wrote a report considering the possibility of realizing $\mu$ catalysis of a $\mathrm{D}+\mathrm{D}$ reaction on a macroscopic scale with a positive energy balance, and I made some calculations. $^{15}$

Some years later, but still before the experimental discovery, Yakov Zel'dovich considered some aspects of the muon-catalysis process. ${ }^{16}$ As well as discussing reactions by $p \mu$ or $d \mu$ atoms in flight, he treated the vibrational states of the molecular ion, estimating for the $d \mu d$ ion the lowest and first excited vibrational binding energies as $\sim 330$ and $\sim 30 \mathrm{eV}$. He then said (in translation):

The presence of the oscillation level, real or virtual, with an energy very near to zero, can greatly increase the amplitude of the wave function in the hole and at the entrance into the barrier.... Both the probabilities of the reaction in flight and of the reaction in formation of a molecule will be increased. ${ }^{17}$

Zel'dovich acknowledges helpful discussions with Alexandr S. Kompaneets, Lev D. Landau, and Sakharov. It seems clear that theorists in the Soviet Union were 
fully cognizant of the idea of muon-induced catalysis of fusion reactions and had done some serious thinking about it by the end of 1953 .

Notes that I added in proof to my paper acknowledge the work of Frank and Zel'dovich, thanks to the referees. ${ }^{18}$ We were still unaware of Sakharov's 1948 report. Indeed, the interest of Soviet physicists, both theoretical and experimental, continued and for the better part of two decades research in $\mu \mathrm{CF}$ centered in the Soviet Union. The names of Semen Gershtein and Leonid Ponomarev, to name only two of the theoretical "chiefs" who followed Sakharov and Zel'dovich, and of Dzhelepov as an experimental "chief," are enough to remind us of the main currents of early progress. In 1964, Dzhelepov and colleagues' diffusion-chamber results, which indicated a greatly increased cross section for $d \mu d$ molecular formation at $240 \mathrm{~K}$ compared to the Carnegie Tech result at liquid-hydrogen temperatures, gave the first inkling of exciting phenomena waiting to be explored. ${ }^{19}$ Gershtein's unpublished 1958 dissertation had sharpened the theoretical expectations of the enhancement of molecular-formation rates that Zel'dovich had suggested by predicting the $(1,1)$ excited state of the $d \mu t$ molecular ion at less than $10 \mathrm{eV}$ below threshold. E. A. Vesman, a student of Gershtein, made the idea concrete by pointing out the efficacy of the transfer of energy into vibrations of the electronic molecule for small energy releases. ${ }^{20}$

The Dubna experiments continued, ${ }^{21}$ and as is well known, in 1977 Ponomarev and colleagues seized the theoretical initiative. In a series of massive calculations with ever increasing accuracy, they determined the energy levels of the molecular ions formed by a muon and two of the isotopes of hydrogen. ${ }^{22}$ Astoundingly, Zel'dovich's and Vesman's hope was realized in spectacular fashion for the $d \mu d$ and $d \mu t$ systems. Excited muonic molecular states were found, not within 10 or $100 \mathrm{eV}$ of threshold, but within 2.0 and $0.7 \mathrm{eV} .^{23}$ These are electronic, not muonic, energies and permit the delicate interplay of the muonic with the electronic molecular vibrational and rotational excitations and give rise to the striking temperature dependence of the overall rate of $d \mu d$ fusion, for example. Hyperfine interactions play an important role. Families of narrow resonances at thermal and epithermal energies give formation rates a hundred or more times the muon decay rate. The prospect of tens, even hundreds, of catalytic acts by each muon was at hand! The best calculated sticking probabilities are somewhat smaller than my original estimates. For the ${ }^{3} \mathrm{He}$ channel in $d \mu d$, it is $13 \%$ compared to my $15 \%$ (inclusion of the mirror reaction reduces the effective value to $8 \%$ ). For $d \mu t$, the sticking is now $0.6 \%$ compared to my $0.94 \%$, because of improvements beyond the adiabatic approximation and also a somewhat larger estimate of stripping after capture. Experimentally, $d \mu t$ sticking is measured as $\omega_{s}=(0.57 \pm 0.04) \% .^{24}$

The theoretical results on the energy levels, soon supported by Soviet experiments, caused a worldwide reawakening of interest in muon-catalyzed fusion. Accompanying the experimental efforts at laboratories all over the world, theorists calculated the fundamental processes of muonic atom and molecule formation, highly accurate molecular-energy levels, the effects of hyperfine 
structure, scattering of muonic atoms, fusion inside the molecules and in flight, sticking and stripping, and kinetics. The effort for such a specialized field has been prodigious, especially in the last 30 years. On the applied side, ideas continue on how to increase the number of fusions per muon and design hybrid systems to get into the realm of net energy production.

For comparison with the immensely detailed picture we now have of $\mu \mathrm{CF}$, I reproduce in figure 4 an old drawing of mine from 1957, which shows the essentials of the catalytic cycle, as it was then understood. Totally absent in the figure are the

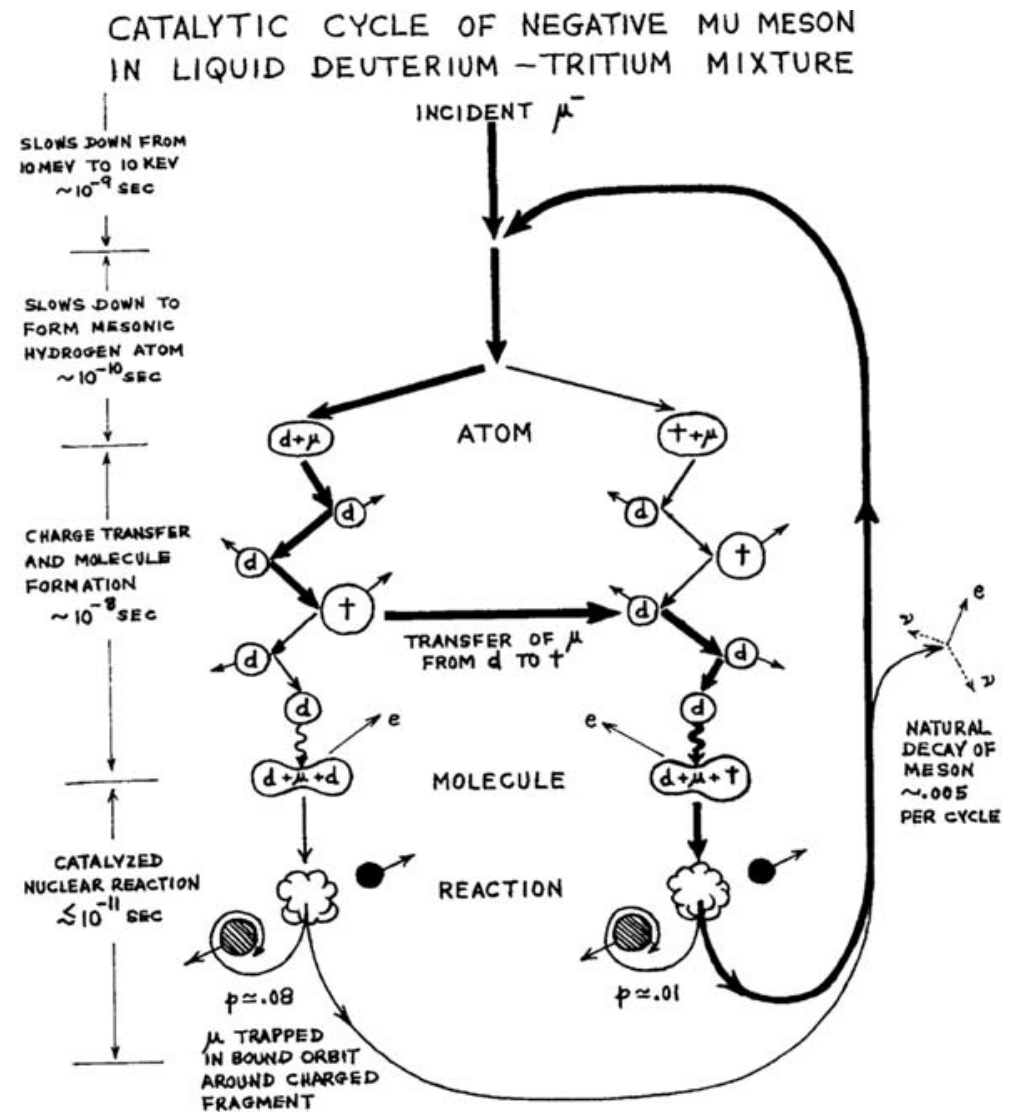

Fig. 4. Sketch of muon-catalyzed fusion cycle for a $D_{2}-T_{2}$ mixture, used in colloquia, $1957 .{ }^{\odot} \mathrm{J}$. D. Jackson. Illustrated are the steps described earlier of the negative muon $\left(\mu^{-}\right)$slowing down from $\mathrm{MeV}$ energies, then sometimes forming a neutral $\left(d^{+} \mu^{-}\right)$atom, often being transferred from the deuteron $(d)$ to a heavier triton $(t)$, with branchings showing a $\left(d^{+} \mu^{-}\right)$atom forming a $(d \mu d)$ molecular ion on the left, or a $\left(t^{+} \mu^{-}\right)$atom forming a $(d \mu t)$ molecular ion on the right followed (most of the time) by the fusion reaction and liberation of the muon to start the catalytic cycle again, or (less likely) on the left the termination of the cycle because of capture of the muon by a helium fusion product. 


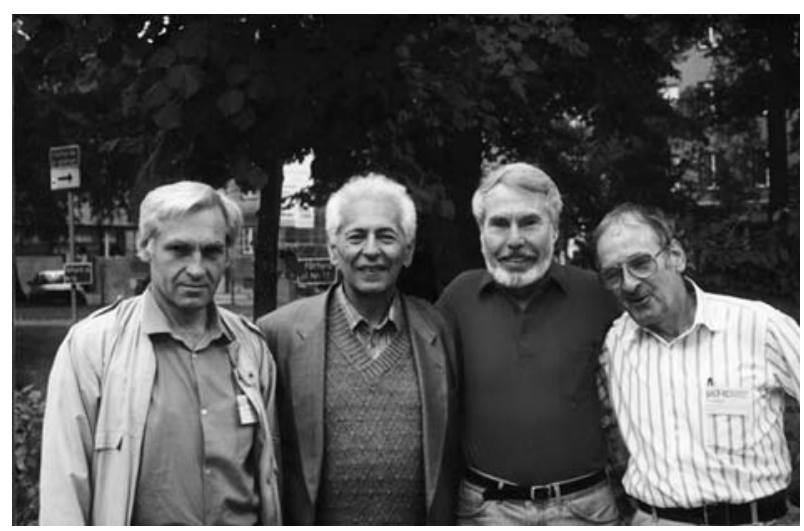

Fig. 5. Left to right Leonid Ponomarev, Semen Gershtein, the author, Yuri Petrov. ${ }^{\circledR} 1992$ J. D. Jackson.

complications (and opportunities) of the hyperfine states, the resonances near threshold, and the crucial Vesman mechanism (only Auger electron emission was envisioned). Nevertheless, we discern the major areas of subsequent research.

I cannot neglect to thank the $\mu \mathrm{CF}$ community for welcoming me into their ranks. My research interests began in theoretical nuclear physics with Viki Weisskopf, then spread into atomic collisions, a bit of plasma physics, but have been in particle physics for most of my career. My one significant shot in $\mu \mathrm{CF}$ occurred long ago. I have watched the phenomenal growth of the subject over the years, but as an outsider. At the Jackson Hole workshop in 1984 and at the $\mu$ CF meeting in Uppsala in 1992 I was privileged to meet many of the theorists and experimenters. I count them all as friends. Among them are the three "Mu-sketers," shown in figure 5 with me at a $\mu \mathrm{CF}$ conference in Uppsala, Sweden, in July 1992.

\section{Acknowledgments}

My recollections here draw on two previous publications. I thank The University of Chicago Press for permission to quote parts of my essay, "Muon Catalysis of Fusion," 25 and Kluwer Academic Publishers for permission to quote parts of my article, "Opening remarks: $\mu \mathrm{CF}$, then and now." 26

Open Access This article is distributed under the terms of the Creative Commons Attribution Noncommercial License which permits any noncommercial use, distribution, and reproduction in any medium, provided the original author(s) and source are credited.

\section{References}

${ }^{1}$ L.W. Alvarez, et al., "Catalysis of Nuclear Reactions by $\mu$ Mesons," Physical Review 105 (1957), $1127-1128$. 
${ }^{2}$ F.C. Frank, "Hypothetical Alternative Energy Sources for the 'Second Meson' Events," Nature 160 (1947), 525-527.

${ }^{3}$ A. D. Sakharov, "Passive Mesons [in Russian]," Report, Academy of Sciences USSR, P.N. Lebedev Physics Institute (April 1948, unpublished); translated into English in Muon Catalyzed Fusion 4 (1989), 235-239; see also S.S. Gerstein and L.I. Ponomarev, "Forty Years Later: Comment on the A.D.S. Report," ibid., 241-245.

${ }^{4}$ Ya. B. Zel'dovich, "Reactions Caused by $\mu$-Mesons in Hydrogen [in Russian]," Doklady Akademii Nauk SSSR 95 (1954), 493-496.

${ }^{5}$ The New York Times, (December 29, 1956), CVI No. 36, B4, 1.

${ }^{6}$ Ibid.

${ }^{7}$ A.S. Wightman, "Moderaton of Negative Mesons in Hydrogen I: Moderation from High Energies to Capture by an $\mathrm{H}_{2}$ Molecule, Phys. Rev. 77 (1950), 521-528.

${ }^{8}$ Leonard I. Schiff, Quantum Mechanics, Third Edition (New York: McGraw-Hill, 1968), p. 292.

9 J.D. Jackson, "Catalysis of Nuclear Reactions between Hydrogen Isotopes by $\mu^{-}$Mesons," Phys. Rev. 106 (1957), 330-339.

${ }^{10}$ Luis W. Alvarez, "Recent developments in particle physics" [Nobel Lecture, December 11, 1968], in Nobel Foundation, Nobel Lectures in Physics 1963-1970 (Amsterdam: Elsevier Publishing Company, 1972; reprinted Singapore: World Scientific, 1998), pp. 241-290; especially pp. 270-272.

${ }^{11}$ Ibid., p. 269.

${ }^{12}$ Frank, "Hypothetical Alternative" (ref. 2).

${ }^{13}$ C.M.G. Lattes, G.P.S. Occhialini, and C.F. Powell, "A Determination of the Ratio of the Masses of $\pi$ - and $\pi$-Mesons by the Method of Grain-counting," The Proceedings of the Physical Society 61 (1948), 173-183.

${ }^{14}$ Sakharov, "Passive Mesons" (ref. 3).

${ }^{15}$ A.D. Sakharov, "Commentary," in D. ter Haar, D.V. Chudnovsky, and G.V. Chudnovsky, ed., Academician Andrei Dmitrievich Sakharov Collected Scientific Works (New York and Basel: Marcel Dekker, 1982), p. 3.

${ }^{16}$ Zel'dovich, "Reactions" (ref. 4).

${ }^{17}$ Ibid., p. 495.

${ }^{18}$ Jackson, "Catalysis of Nuclear Reactions" (ref. 9), p. 330, n. 2.

${ }^{19}$ V.P. Dzhelepov, V.V. Filchenkov, M. Friml, Yu.V. Katyshev, V.I. Moskalev, and P.F. Yermolov, "Negative Muon Catalysis of the Nuclear Fusion Reaction $\mathrm{d}+\mathrm{d} \rightarrow{ }^{3} \mathrm{He}+\mathrm{n}$," Nuovo Cimento 33 (1964), 40-44; idem [Title in Russian], Zhurnal éksperimental'noľ i teoreticheskoř fiziki 46 (1964), 2042-2045.

${ }^{20}$ E.A. Vesman, "Concerning One Possible Mechanism of Production of the Mesic-Molecular Ion (dd $\mu$ ), JETP Letters 5 (1967), 91-93; idem [Title in Russian]," Pis'ma v Zhurnal éksperimental'nǒ i teoreticheskoľ fiziki 5 (1967), 113-115.

${ }^{21}$ For a summary of the Dubna work, see V.V. Filchenkov, "Experimental study of MCF processes at DLNP of JINR," in L.N. Bogdanova, M.P. Faifman, V.I. Korobov, and L.I. Ponomarev, ed., International Conference on Muon Catalyzed Fusion and Related topics ( $\mu$ CF-07) Proceedings (Dubna: Joint Institute for Nuclear Research, 2008), pp. 53-66.

${ }^{22}$ For a personal view of the interplay of theory and experiment, see L. I. Ponomarev, "The History of One Hypothesis," in R.G. Chambers, J.E. Enderby, A. Keller, A.R. Lang, and J.W. Steeds, ed., Sir Charles Frank, OBE, FRS: An eightieth birthday tribute (Bristol, Philadelphia, New 
York: Adam Hilger, 1991), pp. 18-45; idem, "Muon catalyzed fusion," Contemporary Physics 31 (1990), 219-245.

${ }^{23}$ For more recent, extremely accurate computations, see S.A. Alexander and H.J. Monkhorst, "High-accuracy calculation of muonic molecules using random-tempered basis sets," Phys. Rev. A 38 (1988), 26-32.

${ }^{24}$ T. Case, et al., "Systematic analysis of the PSI experiment to directly measure the sticking probability $\omega_{\mathrm{s}}$ in dt fusion" [Proceedings of the International Workshop on Muon Catalyzed fusion ( 295-311.

${ }^{25}$ J. David Jackson, "Muon Catalysis of Fusion," in W. Peter Trower, ed., Discovering Alvarez: Selected Works of Luis W. Alvarez, with Commentary by His Students and Colleagues. (Chicago and London: The University of Chicago Press, 1987), pp. 154-160.

${ }^{26}$ J. D. Jackson, "Opening remarks: $\mu \mathrm{CF}$, then and now" [Proc. Inter. Workshop on Muon Catalyzed Fusion (ref. 24)], Hyperfine Interactions 82 (1993), 3-11.

Department of Physics,

University of California, Berkeley

Berkeley, CA 94720, USA

e-mail: jdjackson@lbl.gov 